\title{
Tratamiento endodóntico en una sesión comparado con múltiples sesiones en pacientes con dentición permanente.
}

\author{
Single versus multiple visits for endodontic treatment in \\ permanent teeth.
}

\author{
Gonzalo Baeza Palacios ${ }^{1,2}$, Karla Morales Puché ${ }^{1,2}$, Ana María Palma Eyzaguirre ${ }^{1,2,3^{*}}$
}

\begin{abstract}
1. Escuela de Odontología, Facultad de Medicina, Pontificia Universidad Católica de Chile, Santiago, Chile

2. Proyecto Epistemonikos, Santiago, Chile

3. Centro de Educación Médica y Ciencias de la

Salud, Facultad de Medicina, Pontificia Universidad

Católica de Chile, Santiago, Chile
\end{abstract}

* Correspondencia Autor: Ana María Palma Eyzaguirre | Dirección: Centro Evidencia UC, Pontificia Universidad Católica de Chile, Diagonal Paraguay 476, Santiago, Chile | E-mail: apalmay@ uc.cl

\section{RESUMEN}

Introducción: El tratamiento endodóntico corresponde a un procedimiento común en la práctica odontológica y su éxito depende de una serie de factores, entre los cuales se ha planteado que existe una diferencia entre los resultados de los tratamientos realizados en una o múltiples visitas. Métodos: Realizamos una búsqueda en Epistemonikos, la mayor base de datos de revisiones sistemáticas en salud, la cual es mantenida mediante el cribado de múltiples fuentes de información, incluyendo MEDLINE, EMBASE, Cochrane, entre otras. Extrajimos los datos desde las revisiones identificadas, analizamos los datos de los estudios primarios, realizamos un meta análisis y preparamos una tabla de resumen de los resultados utilizando el método GRADE. Resultados y conclusiones: Identificamos 10 revisiones sistemáticas que en conjunto incluyeron 46 estudios primarios, de los cuales, 26 corresponden a ensayos aleatorizados que evalúan el tratamiento endodóntico en dientes vitales y no vitales. Concluimos que el tratamiento endodóntico en una sesión podría resultar en poca o nula diferencia sobre el dolor a corto plazo e infecciones postoperatorias, pero la certeza de la evidencia es baja. Además, podría reducir levemente el riesgo de exodoncias dentarias, también podría aumentar el uso de analgésicos y flareups, pero la certeza de la evidencia es baja. Finalmente, no está claro el efecto del tratamiento endodóntico en una sesión sobre el dolor a mediano plazo, debido a que la certeza de la evidencia existente ha sido evaluada como muy baja.

\begin{abstract}
Introduction: Endodontic treatment is a common procedure in dentistry and its success depends on several factors. It has been suggested that there is a difference in terms of effectiveness on endodontic treatments performed in one compared to those performed in multiple visits. Methods: We searched in Epistemonikos, the largest database of systematic reviews in health, which is maintained by screening multiple information sources, including MEDLINE, EMBASE, Cochrane, among others. We extracted data from the systematic reviews, reanalyzed data of primary studies, conducted a meta-analysis and generated a summary of findings table using the GRADE approach. Results and conclusions: We identified 10 systematic reviews including 46 studies overall, of which 26 were randomized trials. We concluded that single versus multiple visits for endodontic treatment in permanent teeth may make little or no difference to short-term pain and postoperative infection, but the certainty of the evidence has been assessed as low. Also, single visit treatment may reduce the risk of extraction due to endodontic problem, increased the risk of painkiller use and flare-ups, but the certainty of the evidence has been assessed as low. We are uncertain about the effect of single visit treatment on medium-term pain, as the certainty of the evidence has been assessed as very low.
\end{abstract}

\section{KEY WORDS}

Root canal therapy; Office visits; Single-visit; Multiple-visits; Pain, Epistemonikos, GRADE.

Int. J. Inter. Dent Vol. 13(3); 217-223, 2020. 


\section{PROBLEMA}

El tratamiento endodóntico corresponde a un procedimiento común en la práctica odontológica y está indicado en casos en que una injuria como una lesión de caries o trauma altera la salud pulpar de un diente hasta generar una patología de carácter irreversible. Un correcto diagnóstico pulpar permite una planificación terapéutica con el objetivo de mantener el diente en la cavidad oral y prevenir otras complicaciones como dolor postoperatorio, uso de analgésicos, infección o incluso la exodoncia del diente afectado.

El éxito del tratamiento endodóntico depende de diversos factores relacionados con el estado de salud general, condición preoperatoria del diente, así como los procedimientos y técnicas endodónticas utilizadas ${ }^{[1]}$. Uno de los factores más controvertidos respecto al procedimiento es el número de sesiones en las que se debería llevar a cabo el tratamiento, ya que el tiempo involucrado podría afectar la evolución de la patología, posibilidades terapéuticas y sus respectivos pronósticos, además de los costos económicos involucrados (accesibilidad) y adherencia al tratamiento.

Si bien existen enfoques que recomiendan realizar una visita para finalizar una biopulpectomía (dientes vitales) y más de una sesión para una necropulpectomía (dientes no vitales), existe incertidumbre en general sobre los efectos que podría tener el número de sesiones en el éxito del tratamiento endodóntico ${ }^{[2]}$.

En este sentido, y gracias a los avances tecnológicos que han permitido reducir el tiempo operatorio, se ha planteado que realizar el tratamiento en una sesión podría disminuir el riesgo de contaminación microbiana entre sesiones, el trauma gingival, aumentar la adherencia por parte del paciente y disminuir los costos asociados a la intervención $n^{[3]}$. Por otra parte, realizar un tratamiento endodóntico en múltiples sesiones incluiría beneficios como la posibilidad de medicación intracanal, cuyo objetivo es reducir la sintomatología y secundar la desinfección, además de la posibilidad de monitorear la evolución de la patología ${ }^{[3]}$.

Este resumen tiene por objetivo evaluar la efectividad y seguridad del tratamiento endodóntico en una sesión comparado con múltiples sesiones".

\section{MÉTODOS}

Realizamos una búsqueda en Epistemonikos, la mayor base de datos de revisiones sistemáticas en salud, la cual es mantenida mediante búsquedas en múltiples fuentes de información, incluyendo MEDLINE, EMBASE, Cochrane, entre otras. Extrajimos los datos desde las revisiones identificadas y analizamos los datos de los estudios primarios. Con esta información, generamos un resumen estructurado denominado FRISBEE (Friendly Summaries of Body of Evidence using Epistemonikos), siguiendo un formato preestablecido, que incluye mensajes clave, un resumen del conjunto de evidencia (presentado como matriz de evidencia en Epistemonikos), metanálisis del total de los estudios cuando sea posible, una tabla de resumen de resultados con el método GRADE y una sección de otras consideraciones para la toma de decisión.

\section{Mensajes clave}

- Realizar tratamiento endodóntico en una sesión podría resultar en poca o nula diferencia sobre el dolor postoperatorio a corto plazo e infección postoperatoria (certeza de la evidencia baja).

- No es posible establecer con claridad si realizar el tratamiento endodóntico en una sesión reduce el dolor a mediano plazo, debido a que la certeza de la evidencia ha sido evaluada como muy baja.

- Realizar tratamiento endodóntico en una sesión podría disminuir levemente el riesgo de exodoncia dentaria del diente tratado (certeza de la evidencia baja).

- Realizar tratamiento endodóntico en una sesión podría aumentar el uso de analgésicos y flare- ups (certeza de la evidencia baja).
Acerca del conjunto de evidencia para esta pregunta

Encontramos 10 revisiones sistemáticas ${ }^{[1],[4],}$

[5], [6], [7], [8], [9], [10], [11], [12] que incluyeron 46 estudios primarios $^{[13],[14],[15],[16],[17],[18],[19],[20], ~[21], ~[22], ~[23], ~[24], ~[25], ~}$ [26], [27], [28], [29], [30], [31], [32], [33], [34], [35], [36], [37], [38], [39], [40], [41], [42], [43], [44], [45], [46], [47], [48], [49], [50], [51], [52], [53], [54], [55], [56], [57], ${ }^{[58]}$, de los cuales 26 corresponden a ensayos Cuál es la $\quad$ aleatorizados [16], [19]], [22], [23], [25], [26], [29], [32]], [34], [35], [37], [38],

evidencia

Véase matriz de evidencia en Epistemonikos más abajo.

Un ensayo ${ }^{[38]}$ se excluyó del análisis dado que incluía exclusivamente a pacientes que requerían retratamiento endodóntico, lo que se consideró una población no comparable a endodoncia primaria.

Esta tabla y el resumen en general se basan en los 25 ensayos restantes [16], [19], [22], [23], [25], [26], [29], [32] [34], [35], [37], [39], [42], [43], [46], [47], [48], [49], [50], [51], [52], [53], [54], [55],

${ }^{[57]}$, dado que los estudios observacionales no aumentaban la certeza de la evidencia existente, ni entregaban información adicional relevante.

Catorce ensayos ${ }^{[19], ~[22], ~[26], ~[32], ~[34], ~[37], ~[39], ~[42], ~[47], ~[48], ~}$ [49], [50], [51], [53] incluyeron pacientes con dentición permanente entre los 12 y 84 años. El resto de los ensayos no reportó la edad de sus pacientes.

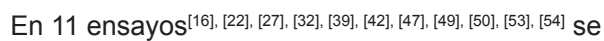
describe el tipo de diente tratado, en los cuales ocho corresponden a dientes uniradiculares ${ }^{[16]}$, [22], [27], [32], [42], [47], [49], [54] y tres correspondían a

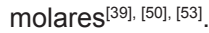

pacientes incluyeron los estudios*

En 17 ensayos [16], [19], [23], [25], [29], [32], [34], [35], [37], [42], [43], [47], [48], [49], [50], [53], [54] se describe el estado pulpar del diente a tratar: dos ensayos sólo incluyeron dientes vitales ${ }^{[23],[32],} 14$ ensayos sólo incluyeron dientes no vitales ${ }^{[19],}$, [25], [29], (34), [35], [37], [42], (43), (47], [48], [49], [50], [53], [54] y un ensayo incluyó ambas opciones ${ }^{[16]}$.

Todos los ensayos compararon la realización del tratamiento endodóntico en una sesión contra tratamiento en múltiples sesiones (dos o más sesiones)

Qué tipo de intervenciones incluyeron los estudios*

Cuatro ensayos compararon contra tratamiento endodóntico en dos sesiones sin medicación intracana[[16], [22], [26], [39] y 11 ensayos evaluaron el tratamiento en dos sesiones [19], [23], [32], [34], [37], [42], [48], [50], [53], [54], [57] con medicación intracanal.

Diez ensayos compararon contra tratamiento endodóntico en tres o más sesiones ${ }^{[25], ~[27], ~[29], ~[35], ~[43], ~}$ [46], [47], [49], [51], [52].

Los ensayos reportaron múltiples desenlaces, los cuales fueron agrupados por las revisiones sistemáticas de la siguiente manera:

- Dolor postoperatorio, medido de manera dicotómica y continua (escala visual análoga)

- Flare- ups

- Inflamación

- Rango de curación

- Infección

Qué tipo de desenlaces midieron

- Uso de analgésicos

- Tasa de éxito radiográfica

- Sensibilidad a la percusión

- Lesión periapical

- Aumento de movilidad dentaria

- Alteración sistémica

- Exodoncia

- Eliminación bacteriana

- Complicaciones postoperatorias

El rango de seguimiento promedio de los ensayos fluctuó entre 6 horas y 5 años.

* La información sobre los estudios primarios es extraída desde las revisiones sistemáticas identificadas, no directamente desde los estudios, a menos que se especifique lo contrario. 


\section{RESUMEN DE LOS RESULTADOS}

La información sobre los efectos de realizar tratamiento endodóntico en una sesión comparado con múltiples sesiones está basada en 17 ensayos aleatorizados que incluyeron 2.941 pacientes (3.047 dientes) $)^{[22]}$. [23], [25], [26], [32], [34], [37], [39], [46], [49], [50], [51], [52], [53], [54], [55], [57]

Con respecto al desenlace del dolor, este fue reportado por los ensayos de forma dicotómica y contínua. En la tabla SoF se reporta en forma dicotómica ya que presenta la mayor cantidad de estudios, considerando que ambos presentan la misma dirección del efecto beneficiando a la intervención.

Nueve ensayos clínicos aleatorizados midieron el desenlace dolor a corto plazo en 1.659 dientes $^{[25], ~[26], ~[32], ~[46], ~[49], ~[50], ~[52], ~[55], ~[57] . ~ S e i s ~ e n s a y o s ~}$ midieron el desenlace dolor postoperatorio a mediano plazo en 990

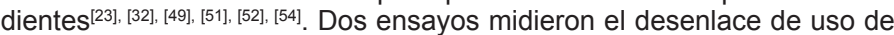
analgésicos en 149 pacientes ${ }^{[32], ~[49] . ~ U n ~ e n s a y o ~ m i d i o ́ ~ e l ~ d e s e n l a c e ~ d e ~}$ exodoncia dentaria en 220 dientes $^{[51]}$. Dos ensayos midieron el desenlace infección en 345 dientes ${ }^{[34], ~[37] ~ y ~ o c h o ~ e n s a y o s ~ m i d i e r o n ~ e l ~ d e s e n l a c e ~}$ flare- ups en 829 dientes $^{[22], ~[25], ~[32], ~[39], ~[49], ~[50], ~[52], ~[53] . ~}$
El resumen de los resultados es el siguiente:

- Realizar tratamiento endodóntico en una sesión podría resultar en poca o nula diferencia en el dolor postoperatorio a corto plazo (certeza de la evidencia baja).

- No es posible establecer con claridad si el tratamiento endodóntico en una sesión reduce el dolor postoperatorio a mediano plazo debido a que la certeza de la evidencia ha sido evaluada como muy baja.

- Realizar tratamiento endodóntico en una sesión podría aumentar el uso de analgésicos post tratamiento (certeza de la evidencia baja).

- Realizar tratamiento endodóntico en una sesión podría disminuir levemente el riesgo de exodoncia dentaria del diente tratado (certeza de la evidencia baja)

- Realizar tratamiento endodóntico en una sesión podría resultar en poca o nula diferencia en el riesgo de infección postoperatoria (certeza de la evidencia baja).

- Realizar tratamiento endodóntico en una sesión podría aumentar el riesgo de aparición de flare- ups (certeza de la evidencia baja).

\begin{tabular}{|c|c|c|c|c|}
\hline \multicolumn{5}{|c|}{ Tratamiento endodóntico en una sesión comparado con múltiples sesiones } \\
\hline $\begin{array}{l}\text { Pacientes } \\
\text { Intervención } \\
\text { Comparación }\end{array}$ & \multicolumn{4}{|c|}{$\begin{array}{l}\text { Pacientes sometidos a tratamiento de endodoncia } \\
\text { Tratamiento en una sesión } \\
\text { Tratamiento en múltiples sesiones }\end{array}$} \\
\hline \multirow{3}{*}{ Desenlaces } & \multicolumn{2}{|c|}{ Efecto absoluto* } & \multirow{3}{*}{$\begin{array}{l}\text { Efecto } \\
\text { relativo } \\
\text { (IC 95\%) }\end{array}$} & \multirow{3}{*}{$\begin{array}{l}\text { Certeza de } \\
\text { la evidencia } \\
\text { (GRADE) }\end{array}$} \\
\hline & $\begin{array}{c}\text { EN } \\
\text { múltiples sesiones }\end{array}$ & $\begin{array}{c}\text { EN } \\
\text { una sesión } \\
\end{array}$ & & \\
\hline & \multicolumn{2}{|c|}{ Diferencia: dientes por 1000} & & \\
\hline \multirow{2}{*}{$\begin{array}{l}\text { Dolor a corto } \\
\text { plazo** }\end{array}$} & 413 por 1000 & 405 por 1000 & \multirow{2}{*}{$\begin{array}{l}\text { RR } 0,98 \\
(0,84 \text { a } \\
1,14)\end{array}$} & \multirow{2}{*}{$\begin{array}{c}\oplus \oplus \bigcirc^{1,2} \\
\text { Baja }\end{array}$} \\
\hline & \multicolumn{2}{|c|}{$\begin{array}{c}\text { Diferencia: } 8 \text { menos } \\
\text { (Margen de error: } 66 \text { menos a } 58 \text { más) }\end{array}$} & & \\
\hline \multirow{2}{*}{$\begin{array}{l}\text { Dolor a mediano } \\
\text { plazo** }\end{array}$} & 139 por 1000 & 228 por 1000 & \multirow{2}{*}{$\begin{array}{l}\text { RR } 1,64 \\
(1,05 \text { a } \\
2,56)\end{array}$} & \multirow{2}{*}{$\begin{array}{l}\oplus \bigcirc \bigcirc \bigcirc^{1,2,3} \\
\text { Muy Baja }\end{array}$} \\
\hline & \multicolumn{2}{|c|}{$\begin{array}{c}\text { Diferencia: } 89 \text { más } \\
\text { (Margen de error: } 7 \text { a } 216 \text { más) }\end{array}$} & & \\
\hline \multirow[b]{2}{*}{$\begin{array}{l}\text { Uso de } \\
\text { analgésicos }\end{array}$} & 92 por 1000 & 121 por 1000 & \multirow{2}{*}{$\begin{array}{l}\operatorname{RR} 1,32 \\
(0,52 \text { a } \\
3,34)\end{array}$} & \multirow[b]{2}{*}{${ }_{\text {Baja }}^{\oplus \oplus \bigcirc}$} \\
\hline & \multicolumn{2}{|c|}{$\begin{array}{l}\text { Diferencia: } 29 \text { pacientes más } \\
\text { (Margen de error: } 44 \text { menos a } 216 \text { más) }\end{array}$} & & \\
\hline \multirow{2}{*}{$\begin{array}{l}\text { Exodoncia } \\
\text { dentaria }\end{array}$} & 19 por 1000 & 8 por 1000 & \multirow{2}{*}{$\begin{array}{l}\text { RR } 0,44 \\
(0,04 \text { a } \\
4,78)\end{array}$} & \multirow{2}{*}{$\begin{array}{c}\oplus \oplus \mathrm{Baja}^{1,2} \\
\mathrm{Ba}\end{array}$} \\
\hline & \multicolumn{2}{|c|}{$\begin{array}{l}\text { Diferencia: } 11 \text { menos } \\
\text { (Margen de error: } 19 \text { menos a } 73 \text { más) }\end{array}$} & & \\
\hline \multirow{2}{*}{ Infección } & 12 por 1000 & 12 por 1000 & $\begin{array}{l}\text { RR } 0,98 \\
(0,15 \text { a }\end{array}$ & $\begin{array}{l}\oplus \oplus \bigcirc^{1,2} \\
\text { Baja }\end{array}$ \\
\hline & \multicolumn{2}{|c|}{$\begin{array}{l}\text { Diferencia: } 0 \text { menos } \\
\text { (Margen de error: } 10 \text { menos a } 66 \text { más) }\end{array}$} & $6,48)$ & \\
\hline \multirow[b]{2}{*}{ Flare- ups*** } & 57 por 1000 & 93 por 1000 & \multirow{2}{*}{$\begin{array}{l}\operatorname{RR} 1,65 \\
(1,03 \text { a } \\
2,63)\end{array}$} & \multirow{2}{*}{$\begin{array}{c}\oplus \oplus \bigcirc^{1,2} \\
\text { Baja }\end{array}$} \\
\hline & \multicolumn{2}{|c|}{$\begin{array}{l}\text { Diferencia: } 36 \text { más } \\
\text { (Margen de error: de } 2 \text { menos a } 92 \text { más) }\end{array}$} & & \\
\hline
\end{tabular}




\section{Margen de error: Intervalo de confianza del 95\% (IC 95\%). \\ RR: Riesgo relativo. \\ GRADE: Grados de evidencia del GRADE Working Group (ver más adelante).}

*Los riesgos EN una sesión están basados en los riesgos del grupo control en los estudios. El riesgo EN múltiples sesiones (y su margen de error) está calculado a partir del efecto relativo (y su margen de error).

** El desenlace dolor a corto plazo fue evaluado en un intervalo de 72 hrs post tratamiento y dolor a mediano plazo fue evaluado a una semana post tratamiento.

*** En el desenlace "Uso de analgésicos" fue el único en el que la unidad de medida fueron pacientes.

**** El desenlace flare- ups fue definido por la presencia de dolor y/o inflamación.

${ }^{1}$ Se disminuyó un nivel de certeza de la evidencia por riesgo de sesgo, ya que se observaron limitaciones asociadas al ocultamiento de la asignación, generación de la secuencia aleatoria y ciego de tratantes y examinadores.

${ }^{2}$ Se disminuyó un nivel de certeza de la evidencia por imprecisión, ya que a cada extremo del intervalo de confianza se tomarían decisiones diferentes.

${ }^{3}$ Se disminuyó un nivel de certeza de la evidencia por inconsistencia, ya que la variabilidad observada en las conclusiones de los estudios no tiene una explicación aparente (I2=57\%).

Siga el enlace para acceder a la versión interactiva de esta tabla (Interactive Summary of Findings - iSoF)

\begin{tabular}{l}
\hline Acerca de la certeza de la evidencia (GRADE)* \\
\hline$\oplus \oplus \oplus \oplus$ \\
Alta: La investigación entrega una muy buena indicación del efecto probable. La probabilidad de \\
que el efecto sea sustancialmente distinto† es baja. \\
$\oplus \oplus \oplus \bigcirc$ \\
Moderada: La investigación entrega una buena indicación del efecto probable. La probabilidad de \\
que el efecto sea sustancialmente distinto† es moderada. \\
$\oplus \oplus \bigcirc$ \\
Baja: La investigación entrega alguna indicación del efecto probable. Sin embargo, la probabilidad \\
de que el efecto sea sustancialmente distinto† es alta. \\
$\oplus \bigcirc \bigcirc \bigcirc$ \\
Muy baja: La investigación no entrega una estimación confiable del efecto probable. La \\
probabilidad de que el efecto sea sustancialmente distinto† es muy alta. \\
\hline *Esto es también denominado 'calidad de la evidencia' o 'confianza en los estimadores del efecto'. \\
$\dagger$ Sustancialmente distinto = una diferencia suficientemente grande como para afectar la decisión \\
\hline
\end{tabular}

\section{OTRAS CONSIDERACIONES PARA LA TOMA DE DECISIÓN}

\section{A quién se aplica y a quién no se aplica esta evidencia}

- Los resultados de este resumen son aplicables a todos los pacientes con necesidad de tratamiento endodóntico en dientes permanentes.

- En los ensayos excluyeron a pacientes con enfermedades sistémicas no controladas, embarazo, retratamiento y uso de analgesia, antibióticos y corticoides. Sin embargo, los resultados del resumen son igualmente aplicables a ellos.

\section{Sobre los desenlaces incluidos en este resumen}

- Los desenlaces sobre dolor postoperatorio a corto y mediano plazo, uso de analgésicos, exodoncia, infección y flare- ups constituyen los outcomes críticos para la toma de decisión debido a que son los que tienen mayor repercusión en la calidad de vida del paciente, ya que son percibidos por ellos.

- Estos desenlaces coinciden en general con los evaluados en las revisiones sistemáticas identificadas.

\section{Balance daño/beneficio y certeza de la evidencia}

- La evidencia muestra que realizar el tratamiento endodóntico en una sesión comparado con múltiples sesiones agregaría un posible beneficio en cuanto a la disminución del riesgo de requerir exodoncia dentaria. Sin embargo, podría aumentar el uso de analgésicos y aparición de flareups, pero la certeza de la evidencia es baja.
- Por otra parte, no habrían diferencias en el desarrollo de dolor a corto plazo y riesgo de infecciones al realizar el tratamiento en una sesión o en más de una sesión, pero la certeza de la evidencia es baja.

- Además, no está claro cuál es el efecto de la intervención en el dolor a mediano plazo, dado que la certeza de la evidencia fue evaluada como muy baja.

- Es importante destacar que a pesar de que los ensayos incluyeron pacientes cuyos dientes tratados variaron en tipo (uniradiculares o molares) y estado pulpar (vitales, no vitales o una mezcla de ambos), sólo se observó inconsistencia en uno de los desenlaces evaluados (dolor a mediano plazo).

- Considerando la incertidumbre existente, no es posible realizar un adecuado balance entre riesgos y beneficios al comparar el tratamiento endodóntico en una o múltiples sesiones. Por lo tanto, la toma de decisión debería considerar las características individualizadas de los pacientes entre las que se encuentran el diagnóstico endodóntico, vitalidad pulpar, historia y complejidad del cuadro, en conjunto a los valores y preferencias de pacientes y tratantes.

\section{Consideraciones de recursos}

- La evidencia analizada no realizó un análisis formal de costos y beneficios comparando el tratamiento endodóntico en una sesión con múltiples sesiones. Sin embargo se menciona la menor demanda de recursos (instrumental y de personal) cuando se realiza el tratamiento en una sesión en comparación con el tratamiento en múltiples sesiones ${ }^{[1], ~[9]}$. 
- Por otro lado, hay evidencia que sugiere que el beneficio de reducir el tiempo de tratamiento a una sesión tanto para el paciente como para el dentista tendría un mayor costo al aumentar la frecuencia del uso de analgésicos postoperatorios, el dolor postoperatorio a mediano plazo y flare ups ${ }^{[1],[8],[11] \text {. }}$

\section{Qué piensan los pacientes y sus tratantes}

- Enfrentados a la evidencia disponible, es probable que exista variabilidad respecto a las preferencias de pacientes y tratantes en el número de sesiones a utilizar, considerando la incertidumbre existente.

- En ocasiones el acceso a la atención en salud es una barrera para los pacientes: la derivación y largos tiempos de espera para obtener el tratamiento dental, sumados al dolor que está asociado a algunas patologías endodónticas, crea una cierta animadversión al tratamiento y a una posible extensión del mismo ${ }^{[59]}$. La mayoría de los pacientes podría mostrar preferencias por el tratamiento endodóntico en una sesión, principalmente porque requiere solo una visita al odontólogo y por consiguiente un menor tiempo de atención, pero esta decisión podría variar considerando los recursos asociados al riesgo de flare-ups, dolor a mediano plazo y un aumento del uso de analgésicos.

- Los odontólogos consideran una serie de factores al elegir el tipo de tratamiento, entre ellos la capacidad del operador y la experiencia clínica, la patología del diente a tratar (si está sintomático o asintomático, presencia o ausencia de inflamación), tiempo de tratamiento adecuado, limitaciones de tiempo del paciente, asistencia médica, historia y actitud, así como consideraciones anatómicas y biológicas ${ }^{[8]}$. En base a estos factores, la mayoría de los tratantes se inclina a favor de realizar tratamientos de dientes vitales (biopulpectomías) en una sesión, mientras que para tratamientos en dientes no vitales (necropulpectomías) pueden presentarse diferentes posturas según la formación del odontólogo, la evolución de la patología y el contexto de la atención.

\section{Diferencias entre este resumen y otras fuentes}

- Las conclusiones de este resumen concuerdan con las 10 revisiones sistemáticas identificadas ${ }^{[1], ~[4], ~[5], ~[6], ~[7], ~[8], ~[9], ~[10], ~[11], ~[12], ~ l a s ~ c u a l e s ~ c o n s i d e r a n ~}$ que no hay evidencia suficiente para demostrar que un tratamiento sea mejor que otro, basado en el nivel de certeza de la evidencia disponible. Así mismo sugieren que el beneficio de reducción del tiempo tanto para el paciente como para el dentista en el tratamiento en una sesión, tiene el costo de una mayor frecuencia en el uso de analgésicos postoperatorios, dolor postoperatorio a mediano plazo y flare- ups.

- Diferentes revisiones de la literatura acerca de tratamientos endodónticos en una o múltiples sesiones, indican que el establecimiento del diagnóstico pulpar y periapical ayuda significativamente a un tratante a decidir una de las dos opciones. Para los dientes vitales un enfoque de una sola visita puede permitir una mayor eficiencia en el cuidado, ya que el sistema de canales se encuentra relativamente no contaminado. Para los dientes diagnosticados con necrosis pulpar, algunos investigadores sugieren que el uso de un medicamento intracanal entre visitas conduce a una mayor desinfección de los canales, mientras que otros no han encontrado diferencias en las tasas de éxito entre los dos enfoques. Sin embargo, para los dientes no vitales sintomáticos y los dientes asintomáticos con lesión periapical, a menudo se ha recomendado el tratamiento con múltiples visitas ${ }^{[60], ~[2], ~[61] . ~}$

- A su vez, las recomendaciones de la guía clínica de endodoncia de la Asociación Americana de Endodoncia[ ${ }^{[62], ~[63], ~[64], ~[65], ~[66], ~[67] ~ i n d i c a n ~ q u e ~}$ los pacientes deben conocer con anticipación que ningún régimen de tratamiento es capaz de prevenir el $100 \%$ de las complicaciones. Se recomienda planificar las sesiones de tratamiento endodóntico según diagnóstico y complejidad.

\section{¿Puede que cambie esta información en el futuro?}

- La probabilidad de que futura investigación cambie las conclusiones de este resumen es alta debido a la incertidumbre de la evidencia existente.

- Identificamos siete ensayos aleatorizados en curso [68], [69], [70], [71], [72], , 773], [74], en la International Clinical Trials Registry Platform de la Organización Mundial de la Salud que podrían arrojar datos relevantes para el desenlace de tratamientos endodónticos realizados en una o más sesiones.

- Identificamos tres revisiones sistemáticas en curso [75], [76], [77], en el International prospective register of systematic reviews (PROSPERO) del National Institute for Health Research.

\section{CÓMO REALIZAMOS ESTE RESUMEN}

Mediante métodos automatizados y colaborativos recopilamos toda la evidencia relevante para la pregunta de interés y la presentamos en una matriz de evidencia.

Siga el enlace para acceder a la versión interactiva:

Tratamiento endodóntico en una sesión comparado con múltiples sesiones.

\section{NOTAS}

Si con posterioridad a la publicación de este resumen se publican nuevas revisiones sistemáticas sobre este tema, en la parte superior de la matriz se mostrará un aviso de "nueva evidencia".

Este artículo es parte del proyecto síntesis de evidencia de Epistemonikos. Se elabora con una metodología preestablecida, siguiendo rigurosos estándares metodológicos y proceso de revisión por pares interno. Cada uno de estos artículos corresponde a un resumen, denominado FRISBEE (Friendly Summary of Body of Evidence using Epistemonikos), cuyo principal objetivo es sintetizar el conjunto de evidencia de una pregunta específica, en un formato amigable a los profesionales clínicos. Sus principales recursos se basan en la matriz de evidencia de Epistemonikos y análisis de resultados usando metodología GRADE. Mayores detalles de los métodos para elaborar este FRISBEE están descritos aquí (http://dx.doi.org/10.5867/medwave.2014.06.5997)

La Fundación Epistemonikos es una organización que busca acercar la información a quienes toman decisiones en salud, mediante el uso de tecnologías. Su principal desarrollo es la base de datos Epistemonikos (www.epistemonikos.org).

\section{DECLARACIÓN DE CONFLICTOS DE INTERESES}

Los autores declaran no tener conflictos de intereses con la materia de este artículo.

\section{AGRADECIMIENTOS}

Este resumen de evidencia fue elaborado con el apoyo metodológico del Centro Evidencia UC, Facultad de Medicina, Pontificia Universidad Católica de Chile.

\section{Bibliografía}

1. Manfredi M, Figini L, Gagliani M, Lodi G. Single versus multiple visits for endodontic treatment of permanent teeth. Cochrane Database Syst Rev. $2016 \mathrm{Dec}$ 1;12(12):CD005296. doi: 10.1002/14651858.CD005296.pub3. PMID: 27905673; PMCID: PMC6463951.

2. Sharma S, Blicher B, Pryles RL, Lin L. Single- Vs Multiple-Visit Root Canal Therapy: Treatment selection requires consideration of factors beyond prognosis. Inside Dent. 2019 Jun; 15(6). Disponible en: https://www.aegisdentalnetwork.com/ id/2019/06/single--vs-multiple-visit-root-canal-therapy

3. Sanjay M, Londhe SM, Sharma S. Single-visit versus dual-visit endodontics. A comparative Study. Indian Journal of Public Health and Development. 2019 July; 10(7). 244 - 250. doi: 10.598.

4. Wong $\mathrm{AW}$, Zhang $\mathrm{C}$, Chu $\mathrm{CH}$. A systematic review of nonsurgical single-visit versus multiple-visit endodontic treatment. Clin Cosmet Investig Dent. 2014 May 8;6:45-56. doi: 10.2147/CCIDE.S61487. PMID: $24855389 ;$ PMCID: PMC4020891. 5. Schwendicke F, Göstemeyer G. Single-visit or multiple-visit root canal treatment: systematic review, meta-analysis and trial sequential analysis. BMJ Open. 2017 Feb 1;7(2):e013115. doi: 10.1136/bmjopen-2016-013115. PMID: 28148534; PMCID: PMC5293988.

6. Sathorn C, Parashos P, Messer H. The prevalence of postoperative pain and flare- up in single- and multiple-visit endodontic treatment: a systematic review. In Endod J. 2008 Feb;41(2):91-9. doi: 10.1111/j.1365-2591.2007.01316.x. Epub 2007 Oct 23. PMID: 17956561.

7. AIRahabi MK. Predictors, prevention, and management of postoperative pain associated with nonsurgical root canal treatment: A systematic review. J Taibah Univ Med Sci. 2017 May 9;12(5):376-384. doi: 10.1016/j.jtumed.2017.03.004. PMID: 31435267; PMCID: PMC6695063.

8. Figini L, Lodi G, Gorni F, Gagliani M. Single versus multiple visits for endodontic treatment of permanent teeth: a Cochrane systematic review. J Endod. 2008 Sep;34(9):1041-7. doi: 10.1016/j.joen.2008.06.009. PMID: 18718362.

9. Su Y, Wang C, Ye L. Healing rate and post-obturation pain of single- versus multiple-visit endodontic treatment for infected root canals: a systematic review. Endod. 2011 Feb;37(2):125-32. doi: 10.1016/j.joen.2010.09.005. Epub 2010 Nov 
12. PMID: 21238790

10. Jorge N.R. Martins, Marina Saura. One appointment endodontic procedure on teeth with apical periodontitis: Is this a criterion for success? - A literature review. Revista Portuguesa de Estomatologia. 2011; 52(3):181-186

11. Sathorn C, Parashos P, Messer HH. Effectiveness of single- versus multiplevisit endodontic treatment of teeth with apical periodontitis: a systematic review and meta-analysis. Int Endod J. 2005 Jun;38(6):347-55. doi: 10.1111/j.13652591.2005.00955.x. PMID: 15910469.

12. Carolina Belén Rosso, Key Fabiano Souza Pereira. Postoperative Pain in Infected Teeth After Single - Versus Multiple-Visit Endodontic Treatment - a Systematic Review. Brazilian Research in Ped Dentistry and Integrated Clinic. 2012; 12(1): 143-148

13. Albashaireh ZS, Alnegrish AS. Postobturation pain after single- and multiplevisit endodontic therapy. A prospective study. J Dent. 1998 Mar;26(3):227-32. doi: 10.1016/s0300-5712(97)00006-7. PMID: 9594474

14. Imura N, Zuolo ML. Factors associated with endodontic flare-ups: a prospective study. Int Endod J. 1995 Sep;28(5):261-5. doi: 10.1111/j.1365-2591.1995.tb00311.x. PMID: 8626209

15. Lin NY, Gao XJ. [A short-term clinical study of one-visit endodontic treatment for infected root canals]. Zhonghua Kou Qiang Yi Xue Za Zhi. 2006 Sep;41(9):525-8. Chinese. PMID: 17129421

16. Patil AA, Joshi SB, Bhagwat SV, Patil SA. Incidence of Postoperative Pain after Single Visit and Two Visit Root Canal Therapy: A Randomized Controlled Trial. J Clin Diagn Res. 2016 May;10(5):ZC09-12. doi: 10.7860/JCDR/2016/16465.7724. Epub 2016 May 1. PMID: 27437339; PMCID: PMC4948515.

17. Yesilsoy C, Koren LZ, Morse DR, Rankow H, Bolanos OR, Furst ML. Postendodontic obturation pain: a comparative evaluation. Quintessence Int. 1988 Jun;19(6):431-8. PMID: 3269592

18. Peters LB, Wesselink PR. Periapical healing of endodontically treated teeth in one and two visits obturated in the presence or absence of detectable microorganisms. Int Endod J. 2002 Aug;35(8):660-7. doi: 10.1046/j.1365-2591.2002.00541.x. PMID: 12196219.

19. Molander A, Warfvinge J, Reit C, Kvist T. Clinical and radiographic evaluation of one- and two-visit endodontic treatment of asymptomatic necrotic teeth with apical periodontitis: a randomized clinical trial. J Endod. 2007 Oct;33(10):1145-8. doi: 10.1016/j.joen.2007.07.005. PMID: 17889679.

20. Prashanth MB, Tavane PN, Abraham S, Chacko L. Comparative evaluation of pain, tenderness and swelling followed by radiographic evaluation of periapica changes at various intervals of time following single and multiple visit endodontic therapy: an in vivo study. J Contemp Dent Pract. 2011 May 1;12(3):187-91. doi: 10.5005/jp-journals-10024-1032. PMID: 22186814

21. Al-Negrish AR, Habahbeh R. Flare up rate related to root canal treatment of asymptomatic pulpally necrotic central incisor teeth in patients attending a military hospital. J Dent. 2006 Oct;34(9):635-40. doi: 10.1016/j.jdent.2005.12.007. Epub 2006 Jan 24. PMID: 16439046.

22. Singh S, Garg A. Incidence of post-operative pain after single visit and multiple visit root canal treatment: A randomized controlled trial. J Conserv Dent. 2012 Oct;15(4):323-7. doi: 10.4103/0972-0707.101888. PMID: 23112477; PMCID: PMC3482743.

23. Gesi A, Hakeberg M, Warfvinge J, Bergenholtz G. Incidence of periapical lesions and clinical symptoms after pulpectomy--a clinical and radiographic evaluation of 1 - versus 2-session treatment. Oral Surg Oral Med Oral Pathol Oral Radiol Endod. 2006 Mar;101(3):379-88. doi: 10.1016/j.tripleo.2005.05.073. PMID: 16504873 24. Walton R, Fouad A. Endodontic interappointment flare-ups: a prospective study of incidence and related factors. J Endod. 1992 Apr;18(4):172-7. doi: 10.1016/ S0099-2399(06)81413-5. PMID: 1402571.

25. Ghoddusi J, Javidi M, Zarrabi MH, Bagheri H. Flare-ups incidence and severity after using calcium hydroxide as intracanal dressing. N Y State Dent J. 2006 JunJul;72(4):24-8. PMID: 16925009.

26. Ince B, Ercan E, Dalli M, Dulgergil CT, Zorba YO, Colak H. Incidence of postoperative pain after single- and multi-visit endodontic treatment in teeth with vital and non-vital pulp. Eur J Dent. 2009 Oct;3(4):273-9. PMID: 19826598; PMCID: PMC2761157.

27. Fava LR. A comparison of one versus two appointment endodontic therapy in teeth with non-vital pulps. Int Endod J. 1989 Jul;22(4):179-83. doi: 10.1111/j.13652591.1989.tb00921.x. PMID: 2637223.

28. Oliet S. Single-visit endodontics: a clinical study. J Endod. 1983 Apr;9(4):147-52. doi: 10.1016/S0099-2399(83)80036-3. PMID: 6574201

29. Waltimo T, Trope M, Haapasalo M, Ørstavik D. Clinical efficacy of treatment procedures in endodontic infection control and one year follow-up of periapical healing J Endod. 2005 Dec;31(12):863-6. doi: 10.1097/01.don.0000164856.27920.85. PMID: 16306819

30. Fava LR. A clinical evaluation of one and two-appointment root canal therapy using calcium hydroxide. Int Endod J. 1994 Jan;27(1):47-51. doi: 10.1111/j.13652591.1994.tb00229.x. PMID: 7806412.

31. Roane JB, Dryden JA, Grimes EW. Incidence of postoperative pain after singleand multiple-visit endodontic procedures. Oral Surg Oral Med Oral Pathol. 1983 Jan;55(1):68-72. doi: 10.1016/0030-4220(83)90308-0. PMID: 6572350.

32. Wang C, Xu P, Ren L, Dong G, Ye L. Comparison of post-obturation pain experience following one-visit and two-visit root canal treatment on teeth with vital pulps: a randomized controlled trial. Int Endod J. 2010 Aug;43(8):692-7. doi: 10.1111/j.1365-2591.2010.01748.x. Epub 2010 May 19. PMID: 20491987

33. Soltanoff W. A comparative study of the single-visit and the multiple-visit edodontic procedure. J Endod. 1978 Sep;4(9):278-81. doi: 10.1016/s0099-2399(78)80144-7. PMID: 283193

34. Penesis VA, Fitzgerald PI, Fayad MI, Wenckus CS, BeGole EA, Johnson BR Outcome of one-visit and two-visit endodontic treatment of necrotic teeth with apica periodontitis: a randomized controlled trial with one-year evaluation. J Endod. 2008
Mar;34(3):251-7. doi: 10.1016/j.joen.2007.12.015. PMID: 18291270.

35. Kvist T, Molander A, Dahlén G, Reit C. Microbiological evaluation of one- and twovisit endodontic treatment of teeth with apical periodontitis: a randomized, clinica trial. J Endod. 2004 Aug;30(8):572-6. doi: 10.1097/01.don.0000121607.87969.6e. PMID: 15273638.

36. Rudner WL, Oliet S. Single-visit endodontics: a concept and a clinical study. Compend Contin Educ Dent. 1981 Mar-Apr;2(2):63-8. PMID: 6950852.

37. Paredes-Vieyra J, Enriquez FJ. Success rate of single- versus two-visit root canal treatment of teeth with apical periodontitis: a randomized controlled trial. J Endod. 2012 Sep;38(9):1164-9. doi: 10.1016/j.joen.2012.05.021. Epub 2012 Jul 26. PMID: 22892729.

38. Yoldas O, Topuz A, Isçi AS, Oztunc H. Postoperative pain after endodontic retreatment: single- versus two-visit treatment. Oral Surg Oral Med Oral Patho Oral Radiol Endod. 2004 Oct;98(4):483-7. doi: 10.1016/j.tripleo.2004.03.009. PMID: 15472665

39. DiRenzo A, Gresla T, Johnson BR, Rogers M, Tucker D, BeGole EA Postoperative pain after 1- and 2-visit root canal therapy. Oral Surg Oral Med Oral Pathol Oral Radiol Endod. 2002 May;93(5):605-10. doi: 10.1067/moe.2002.121900. PMID: 12075212.

40. Eleazer PD, Eleazer KR. Flare-up rate in pulpally necrotic molars in one-visit versus two-visit endodontic treatment. J Endod. 1998 Sep;24(9):614-6. doi: 10.1016/ S0099-2399(98)80122-2. PMID: 9922752.

41. Rao KN, Kandaswamy R, Umashetty G, Rathore VP, Hotkar C, Patil BS. PostObturation pain following one-visit and two-visit root canal treatment in necrotic anterior teeth. J Int Oral Health. 2014 Apr;6(2):28-32. Epub 2014 Apr 26. PMID: 24876699; PMCID: PMC4037792.

42. Dorasani G, Madhusudhana K, Chinni SK. Clinical and radiographic evaluation of single-visit and multi-visit endodontic treatment of teeth with periapical pathology: An in vivo study. J Conserv Dent. 2013 Nov;16(6):484-8. doi: 10.4103/09720707.120933. PMID: 24347878; PMCID: PMC3842712.

43. Xavier AC, Martinho FC, Chung A, Oliveira LD, Jorge AO, Valera MC, Carvalho $\mathrm{CA}$. One-visit versus two-visit root canal treatment: effectiveness in the removal of endotoxins and cultivable bacteria. J Endod. 2013 Aug;39(8):959-64. doi: 10.1016/j. joen.2013.04.027. Epub 2013 May 22. PMID: 23880258.

44. El Mubarak AH, Abu-bakr NH, Ibrahim YE. Postoperative pain in multiple-visit and single-visit root canal treatment. J Endod. 2010 Jan;36(1):36-9. doi: 10.1016/j oen.2009.09.003. PMID: 20003932.

45. Ng YL, Glennon JP, Setchell DJ, Gulabivala K. Prevalence of and factors affecting post-obturation pain in patients undergoing root canal treatment. Int Endod J. 2004 Jun;37(6):381-91. doi: 10.1111/j.1365-2591.2004.00820.x. PMID: 15186245.

46. Pekruhn RB. Single-visit endodontic therapy: a preliminary clinical study. J Am Dent Assoc. 1981 Dec;103(6):875-7. doi: 10.14219/jada.archive.1981.0400. PMID: 6947006.

47. Trope M, Delano EO, Orstavik D. Endodontic treatment of teeth with apica periodontitis: single vs. multivisit treatment. J Endod. 1999 May;25(5):345-50. doi: 10.1016/S0099-2399(06)81169-6. PMID: 10530259.

48. Weiger R, Rosendahl R, Löst C. Influence of calcium hydroxide intracanal dressings on the prognosis of teeth with endodontically induced periapical lesions. Int Endod J. 2000 May;33(3):219-26. doi: 10.1046/j.1365-2591.1999.00298.x. PMID: 11307438

49. Mulhern JM, Patterson SS, Newton CW, Ringel AM. Incidence of postoperative pain after one-appointment endodontic treatment of asymptomatic pulpal necrosis in single-rooted teeth. J Endod. 1982 Aug;8(8):370-5. doi: 10.1016/s00992399(82)80197-0. PMID: 6958790.

50. Risso PA, Cunha AJ, Araujo MC, Luiz RR. Postobturation pain and associated factors in adolescent patients undergoing one- and two-visit root canal treatment. $J$ Dent. 2008 Nov;36(11):928-34. doi: 10.1016/j.jdent.2008.07.006. Epub 2008 Sep 3. PMID: 18771838.

51. Wong AW, Tsang CS, Zhang S, Li KY, Zhang $\mathrm{C}$, Chu $\mathrm{CH}$. Treatment outcomes of single-visit versus multiple-visit non-surgical endodontic therapy: a randomised clinical trial. BMC Oral Health. 2015 Dec 19;15:162. doi: 10.1186/s12903-0150148-x. PMID: 26687126; PMCID: PMC4684923.

52. Oginni A, Udoye $\mathrm{Cl}$. Endodontic flare-ups: comparison of incidence between single and multiple visits procedures in patients attending a Nigerian teaching hospital. Odontostomatol Trop. 2004 Dec;27(108):23-7. PMID: 15853274

53. Akbar I, lqbal A, Al-Omiri MK. Flare-up rate in molars with periapical radiolucency in one-visit vs two-visit endodontic treatment. J Contemp Dent Pract. 2013 May 1;14(3):414-8. doi: 10.5005/jp-journals-10024-1337. PMID: 24171982.

54. Xiao D, Zhang DH. [A clinical study of one-visit endodontic treatment for infected root canals]. Hua Xi Kou Qiang Yi Xue Za Zhi. 2010 Feb;28(1):57-60. Chinese. PMID: 20337077

55. S. Liu, Z.-Y. Leng. Short-term clinical effect of single-visit root canal therapy for infected root canals. Journal of Shanghai Jiaotong University. July 2013; 33(7):1014 1017

56. O'Keefe EM. Pain in endodontic therapy: preliminary study. J Endod. 1976 Oct;2(10):315-9. doi: 10.1016/S0099-2399(76)80047-7. PMID: 1068208.

57. Wong AW, Zhang S, Li SK, Zhu X, Zhang C, Chu CH. Incidence of post-obturation pain after single-visit versus multiple-visit non-surgical endodontic treatments. BMC Oral Health. 2015 Aug 14;15:96. doi: 10.1186/s12903-015-0082-y. PMID: 26272704 PMCID: PMC4536791.

58. Jabeen S, Khurshiduzzaman M. Incidence of post obturation pain following single and multi visit root canal treatment in a teaching hospital of Bangladesh Mymensingh Med J. 2014 Apr;23(2):254-60. PMID: 24858151

59. Melgaço-Costa JL, Martins RC, Ferreira EF, Sobrinho AP. Patients' Perceptions of Endodontic Treatment as Part of Public Health Services: A Qualitative Study. Int J Environ Res Public Health. 2016 Apr 27;13(5):450. doi: 10.3390/ijerph13050450. PMID: 27128932; PMCID: PMC4881075.

60. Lin LM, Lin J, Rosenberg PA. One-appointment endodontic therapy: biological 
considerations. J Am Dent Assoc. 2007 Nov;138(11):1456-62. doi: 10.14219/jada. archive.2007.0081. PMID: 17974642.

61. Kaufmann, R. Single Appointment Endodontics Yes or No? [Internet]. Winnipeg: The EndoExperience [cited 2020 Nov 11]. Available from: https:// www.endoexperience.com/filecabinet/Clinical\%20Endodontics/Single \%20 Appointment $\% 20$ Treatment/Single\%20Appointment $\% 20$ Endodontics $\% 20 \% 20$ Yes\%20or\%20No.pdf

62. American Association of Endodontists. Guide to Clinical Endodontics. American Association of Endodontists. 2013. Available from: https://www.aae.org/specialty/ clinical-resources/guide-clinical-endodontics/

63. American Association of Endodontists. Treatment options for the compromised Tooth: A decision guide. 2017. Available from: https://f3f142zs0k2w1kg84k5p9i1owpengine.netdna-ssl.com/specialty/wp-content/uploads/sites/2/2017/06/2014treat mentoptionsguidefinalweb.pdf

64. American Association of Endodontists. The Treatment of Traumatic Dental Injuries. American Association of Endodontists. 2019. Available from: https:// f3f142zs0k2w1kg84k5p9i1o-wpengine.netdna-ssl.com/specialty/wp-content/ uploads/sites/2/2019/02/19_TraumaGuidelines.pdf

65. American Association of Endodontists. Endodontic Competency. American Association of Endodontists. 2017. Available from: https:// f3f142zs0k2w1 kg84k5p9i1o-wpengine.netdna-ssl.com/specialty/wp-content/ uploads/sites/2/2017/09/endo-competency-whitepaper.pdf

66. American Association of Endodontists Scope of Endodontics: Regenerative Endodontics. American Association of Endodontists. 2018. Available from: https:// f3f142zs0k2w1kg84k5p9i1o-wpengine.netdna-ssl.com/specialty/wp-content/ uploads/sites/2/2018/07/2018AAE_Scope_of_Endo_Regenerative_Endodontics. pdf

67. American Association of Endodontists. Treatment Standards. American Association of Endodontists 2018. Available from: https://f3f142zs0k2w1kg84k5p9i1owpengine.netdna-ssl.com/specialty/wp-content/uploads/sites/2/2018/04/ TreatmentStandards Whitepaper.pdf

68. Kumar Rajendra. Assessment of Clinical and Patient Centered Outcomes of Single Visit versus Multiple Visits Endodontic Treatment. [International Clinica Trials Registry Platform] CTRI/2019/08/020956. Available from: https://apps.who.int trialsearch/Trial2.aspx?TriallD=CTRI/2019/08/020956
69. Nishi Kumari. Incidence of postoperative pain after single visit and multiple visit root canal therapy: A Randomized Controlled Trial. [International Clinical Trials Registry Platform] CTRI/2019/05/019067. Available from: https://apps.who.int/ trialsearch/Trial2.aspx?TriallD=CTRI/2019/05/019067

70. Patrícia A Risso. Periapical Healing After One or Two-visits to Endodontic Treatment in Adolescents Patients. [International Clinical Trials Registry Platform] NCT01312194. Available from: http://clinicaltrials.gov/show/NCT01312194

71. Chu Chun Hung. Treatment outcome of single-visit versus multiple-visit nonsurgical endodontic therapy. [International Clinical Trials Registry Platform] ChiCTRIOR-15006117 . http://www.chictr.org.cn/showproj.aspx?proj=10605.

72. Tosatto Pinto. Single-visit versus multiple-visit endodontic treatment of anterior upper primary teeth with calcium hydroxide as intracanal dressing: Randomized clinical trial. [International Clinical Trials Registry Platform] ISRCTN16209990 http://isrctn.com/ISRCTN16209990

73. XX Healing Rates After Single Versus Multiple Visits Endodontic Treatment of Necrotic Teeth With Apical Periodontitis: A Randomized Clinical Trial. [International Clinical Trials Registry Platform] NCT02947763 https://clinicaltrials.gov/show/ NCT02947763

74. Suzana;Cassia. Quantification of endotoxins and its relation with signs and symptoms: single $\mathrm{X}$ multiple-visits - one year follow-up by CBCT analysis. [International Clinical Trials Registry Platform] RBR-5t399r. http://www. ensaiosclinicos.gov.br/rg/RBR-5t399r/

75. Weidong Niu. Postoperative pain after single versus multiple visits for endodontic treatment. [PROSPERO 2017] CRD42017083606 Available from: https://www.crd. york.ac.uk/prospero/display_record.php?ID=CRD42017083606

76. Athar Mohamed. Clinical and radiographic outcomes following single- versus multiple-visit endodontic treatment in patients with non-vital permanent teeth: a systematic review and meta-analysis. [PROSPERO 2018] CRD42018079562 Available from: php?ID=CRD42018079562 https://www.crd.york.ac.uk/prospero/display_record.

77. Frederico Martinho. What is more effective in reducing endotoxin in endodontic infection - multiple sessions or single appointment? A systematic review of clinical studies with meta-analysis. [PROSPERO 2017] CRD42017077160 Available from: https://www.crd.york.ac.uk/prospero/display_record.php?|D=CRD42017077160 